\title{
Asymptomatic multiple intracardiac tuberculomas in a child
}

\author{
Krishnan Rajeshwari, Sandya Gupta, AnandPrakash Dubey, Rani Gera \\ Department of Pediatrics, Maulana Azad Medical College, New Delhi, India
}

\begin{abstract}
Tuberculosis is widely prevalent among children in India, especially in those with coexisting severe malnutrition and immunodeficiency. Uncommon sites for tuberculosis are frequently seen. However, intracardiac tuberculomas are extremely rare in children. To the best of our knowledge, this is the first case report of multiple asymptomatic intracardiac tuberculomas in a child. Most intracardiac tuberculomas cause symptoms, especially in adults. In asymptomatic children non surgical management of intracardiac tuberculomas appears to be a safe treatment option as complete resolution occurs with antituberculous therapy. (Cardiol J 2012; 19, 5: 518-520)
\end{abstract}

Key words: intracardiac tuberculomas, children

\section{Case report}

Tuberculosis is widely prevalent among children in India, especially in those with coexisting severe malnutrition and immunodeficiency. Uncommon sites for tuberculosis are frequently seen. However, intracardiac tuberculomas are extremely rare in children. We report an unusual case of multiple intracardiac tuberculomas in a child.

An eight year-old boy presented with a history of low grade fever and progressive weight loss of one year's duration. There was history of associated cough without expectoration for the same duration. There was no history of palpitations or dyspnea. He had been taken to various private doctors and received multiple courses of antibiotics but no relief was obtained. Six months later, the child developed one episode of generalized tonic clonic seizure lasting 15 min for which carbamazepine was started. On examination in this hospital, the child had anemia, no lymphadenopathy, jaundice or clubbing. Heart rate was $80 \mathrm{bpm}$, regular and blood pressure was $90 / 60 \mathrm{~mm} \mathrm{Hg}$. He was severely malnourished with wasting and stunting with a weight of
$16 \mathrm{~kg}$ and height of $119 \mathrm{~cm}$. He also had microcephaly with a head circumference of $48 \mathrm{~cm}$. Chest examination revealed bilateral occasional crackles. There was no hepatosplenomegaly. Cardiovascular examination was within normal limits. The child also had mental retardation.

Investigations revealed normocytic normochromic anemia ( $\mathrm{Hb} 8.6 \mathrm{~g} / \mathrm{dL})$, total leukocytic count of 10,440 cells per $\mathrm{mL}$, erythrocyte sedimentation rate of $60 \mathrm{~mm}$ in first hour. Kidney function tests and liver function tests were within normal limits. Mantoux test was reactive. Serological testing for HIV infection was negative. Chest X-ray showed cardiomegaly with widening of carina. Bony cage and soft tissue were normal. Infiltrative opacities were seen in the right upper zone and middle zone and a right lamellar effusion was seen. Two-dimensional echocardiography revealed a large mass in the right atrium (RA), left atrium (LA) and left ventricle (LV) with normal valves. Magnetic resonance imaging (MRI) of the heart revealed heterogeneously enhancing thickened myocardium with associated heterogeneously enhancing intraluminal masses in RA, LA and LV minimal pericar-

Address for correspondence: Prof. Krishnan Rajeshwari, Department of Pediatrics, Maulana Azad Medical College, New Delhi 110002, India, tel: 9968604312, e-mail: rajeshwari.dr@gmail.com 


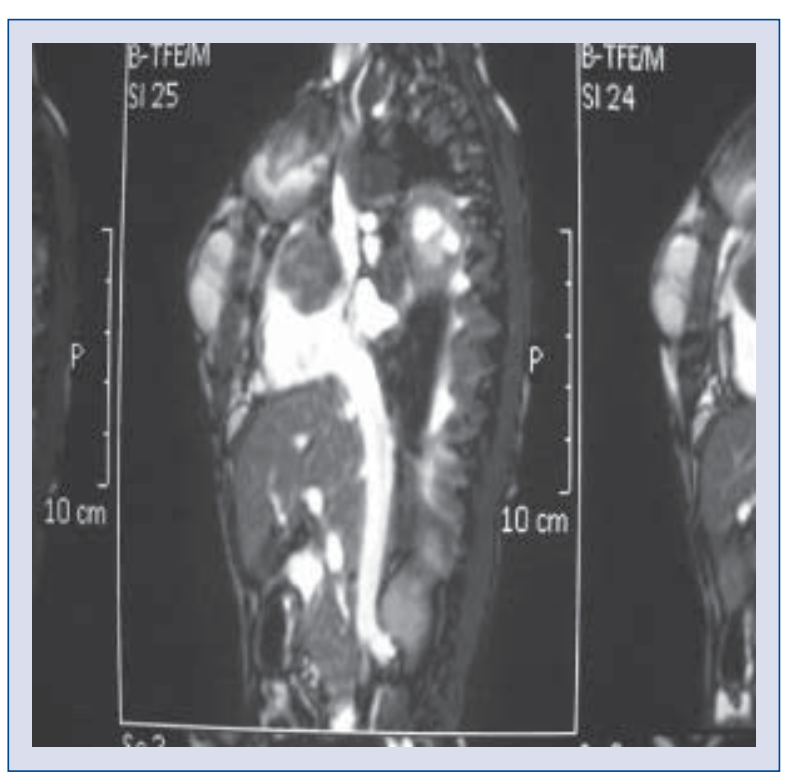

Figure 1. Magnetic resonance imaging of the heart showing heterogeneously enhancing thickened myocardium with associated heterogeneously enhancing intraluminal masses in right and left atrium and left ventricle and minimal pericardial effusion and associated ill defined heterogenously enhancing soft tissue in anterior, middle and posterior mediastinum.

dial effusion and associated ill defined heterogenously enhancing soft tissue in anterior, middle and posterior mediastinum and right paravertebral soft tissue with bony destruction of costovertebral junction and signal alteration in vertebral bodies (Fig. 1). Right sided empyema with minimal left pleural effusion and fibronodular lesions in bilateral lung fields were seen. This MRI was suggestive of pulmonary tuberculosis, with mediastinal involvement along with multiple intracardiac tuberculomas (Fig. 2). Category 1 antitubercular treatment comprising isoniazid, rifampicin, ethambutol and pyrazinamide was started. Fever gradually resolved and the child improved. Repeat MRI done two months later showed that the mass lesions in the atria and LV had decreased in size and anterior, middle and posterior mediastinum showed liquefaction. The sternal and chest wall involvement had increased. The child was subsequently discharged. Fifteen days later, he developed an abscess right chest wall which was diagnosed as empyema necessitans. This was drained and pus was negative for acid fast bacilli stain. Repeat echo done three months after anti tubercular therapy showed a normal heart with resolution of all tuberculomas (Fig. 3). This confirmed the etiology as tubercular, as he had only received antitubercular treatment in the preceding three months.

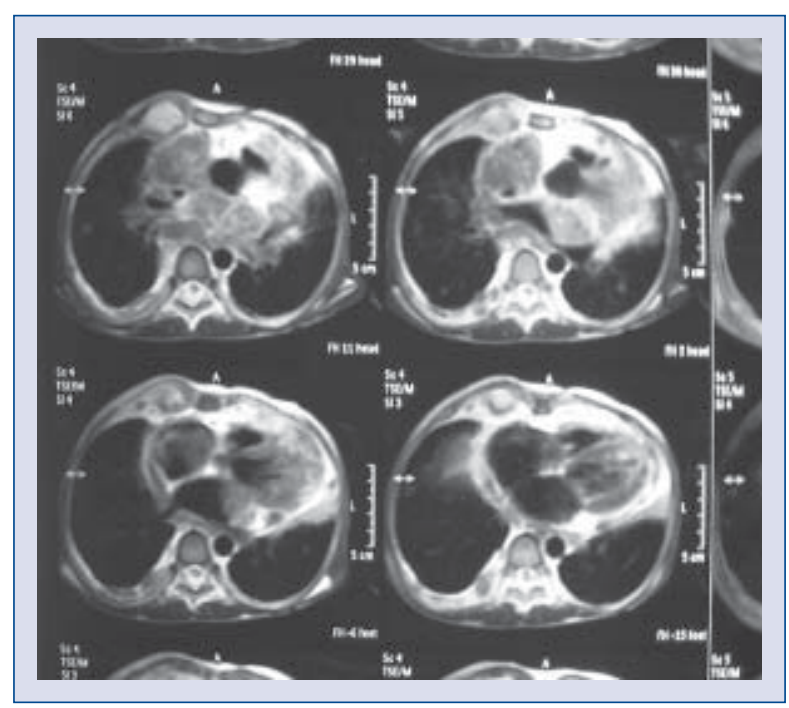

Figure 2. Magnetic resonance imaging was suggestive of pulmonary tuberculosis, with mediastinal involvement along with multiple intracardiac tuberculomas.

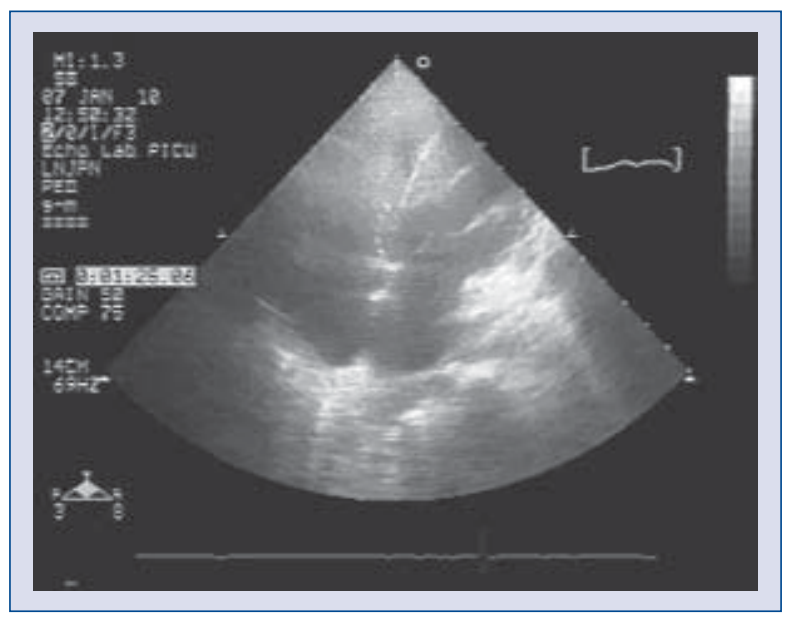

Figure 3. Post treatment echo showing complete resolution of intracardiac tuberculomas.

\section{Discussion}

To the best of our knowledge, this is the first case report of multiple asymptomatic intracardiac tuberculomas in a child.

Among the extrapulmonary manifestations of tuberculosis, involvement of the heart is extremely rare and very infrequently diagnosed ante mortem [1]. In a series of 14,000 deaths due to tuberculosis, involvement of the heart was observed in less than $0.5 \%$ of cases [2]. Three distinct forms are recognized: diffuse infiltrating, miliary, and nodular [3]. 
Although involvement can occur from hematogenous spread, the most likely mechanisms of myocardial involvement are direct retrograde spread from a contiguous mediastinal lymph node or extension from a pericardial focus [4]. This child also had extensive mediastinal and pulmonary tuberculosis. Single or multiple cardiac tuberculomas are most often observed in the right heart chambers, particularly in the wall of the RA. They are usually well circumscribed and sharply demarcated from the surrounding parenchyma. They may erode the underlying myocardium, resulting in ulcers that in turn cause thrombus formation and subsequent embolism. Most clinical reports published have described various symptoms in adults due to cardiac tuberculosis, like ventricular tachycardia $[5,6]$, aortic insufficiency [7], pulmonary vein obstruction caused by LA mass lesion [8], complete heart block [9], congestive heart failure [10], right ventricular outflow tract obstruction [11], and superior vena cava obstruction [12]. This child had no cardiac symptoms at presentation.

Whereas the diagnosis of tuberculosis was almost exclusively made at autopsy in earlier years, advances in imaging techniques give an opportunity for earlier diagnosis. Echocardiography, computed tomography and MRI contribute to an optimal morphologic description and assessment of hemodynamic significance. Cardiac MRI is a recent tool and shows a characteristic $\mathrm{T} 2$ shortening in tuberculous involvement, similar to that seen in intracranial tuberculomas. The characteristic appearance on $\mathrm{T} 2 \mathrm{~W}$ images includes a central isointense core, corresponding to central caseation; a hypointense rim, which represents the fibrous capsule; and a thin hyperintense line, which correlates with an inflammatory cellular infiltrate [13]. This was seen in this case.

To conclude, in areas of high prevalence of tuberculosis in children with extensive chest tuberculosis, multiple cardiac masses should be considered to be tubercular in origin. Histopathological evidence may not always be possible to obtain. MRI of the heart is emerging as a recent important diagnostic tool. Resolution with antitubercular treatment can be demonstrated by serial echocardiography. Most intracardiac tuberculomas cause symptoms, especially in adults. However, even multiple intracardiac masses may be completely asymptomatic, as seen in this child. In asymptomatic children, non surgical management of intracardiac tuberculomas appears to be a safe treatment option.

\section{Acknowledgements}

Dr K. Rajeshwari wrote the manuscript, Dr A.P. Dubey assisted in the drafting and the rest helped in data collection.

\section{Conflict of interest: none declared}

\section{References}

1. Kannangara DW, Salem FA, Rao BS, Thadepalli H. Cardiac tuberculosis: TB of the endocardium. Am J Med Sci, 1984; 287: 45-47.

2. Custer EN, Charr R. Tuberculosis of the myocardium. JAMA, 1939; 112: 1333-1334.

3. Gaultier Y, Alou A, Cenac A, Develoux M, Vetter JM. Tuberculome du Coeur [In French]. Arch Mal Coeur, 1987; 80: 1413-1416.

4. Murtada AH, Mercer EN, Guinn GA. Myocardial tuberculoma with rupture and pseudoaneurysm formation: Successful surgical treatment. Br Heart J, 1985; 54: 603-604.

5. Neill PGO, Rokey R, Greenberg S, Pacifico A. Resolution of ventricular tachycardia and endocardial tuberculoma following antituberculosis therapy. Chest, 1991; 100: 1467-1469.

6. Behr G, Palin HC, Temperley JM. Myocardial tuberculosis. Br Med J, 1977; 1: 951.

7. Soyer R, Brunet A, Chevallier B, Leroy J, Morere M, Redonnet M. Tuberculous aortic insufficiency: Report of a case with successful surgical treatment. J Thorac Cardiovasc Surg, 1981; 82: 254-256.

8. Horn H, Saphir O. The involvement of the myocardium in tuberculosis: A review of literature and report of three cases. Am Rev Tuberc, 1935; 32: 492-506.

9. Wren C, Stovin, PG. Isolated interventricular septal tuberculoma causing complete heart block. Thorax, 1982; 37: 149-150.

10. Krishnaswami H, Cherian G. Right atrial tuberculoma: Report of a case with complete recovery. Thorax, 1984; 39: 550-551.

11. Rawls WJ, Shuford WH, Logan WD, Hurst JW, Schlant RC. Right ventricular outflow tract obstruction produced by a myocardial abscess in a patient with tuberculosis. Am J Cardiol, 1968; 21: 738-745.

12. Kapoor OP, Mascarenhas E, Rananaware MM, Gadgil RK. Tuberculoma of the heart. Report of 9 cases. Am Heart J, 1973; 8693: 334-340.

13. Kim TK, Chang KH, Kim CJ, Goo JM, Kook MC, Han MH. Intracranial tuberculoma: Comparison of $\mathrm{MR}$ with pathologic findings. Am J Neuroradiol, 1995; 16: 1903-1908. 